\title{
The application to the simulation of forest fire spreading based on optimized boundary interpolation
}

\author{
Ding Zhi ${ }^{\mathrm{a}, \mathrm{b}}$ \\ a University of Chinese Academy of Sciences
}

\author{
Liu Dian-wei ${ }^{\mathrm{a}^{*}}$,Wang Zong-ming ${ }^{\mathrm{a}}$,Liu Ying ${ }^{\mathrm{a}, \mathrm{b}}$,Tang \\ Xu-guang ${ }^{\mathrm{a}, \mathrm{b}}$,Dong Zhang-yu ${ }^{\mathrm{a}, \mathrm{b}}$,Jia Ming-ming ${ }^{\mathrm{a}, \mathrm{b}}$,Shao \\ Tian-tian $^{\mathrm{a}, \mathrm{b}}$ \\ b Northeast Institute of Geography and Agroecology, \\ Chinese Academy of Sciences \\ ChangChun 130012,China
}

\begin{abstract}
The boundary interpolation algorithm could quickly get the scope of forest fire spreading and trend of the spreading. However, the result from the algorithm has some problems on precision. Therefore, this article selected the points that was calculated from three adjacent moments as sample points for interpolation, and then had a comparing between the different interpolation methods. The result showed that the optimized boundary interpolation algorithm has more approaches to get the spreading scope and improves the accuracy of the result of the simulation of forest fire spreading. With the algorithm, it will be easier to quickly catch the accurate dynamic information of the forest fire spreading.
\end{abstract}

Key words-simulation of forest fire spreading, boundary interpolation, IDW.

\section{INTRODUCTION}

The forest fire hazard is huge, possessing the characteristic of happening suddenly and being difficult to control. If the dynamic of the forest fire spread can be simulated fast and accurately, it will have a key important role on fire suppression and fire fighting(ZHAO et al., 2010). Various countries have built the forest fire spread model which suits their own conditions, such as Rothermel model of the United States(Rothermel R. C et al., 1972), McArthur model of Australia, national forest fire spreading model of Canada(Bak et al., 1987; Karafyllidis et al., 1997; Barros et al., 1997), and Wang Zhengfei model of China(Tang et al., 2002).. The spreading models proposed by Wang Zhengfei. and its correction model are more extensive applied in China. According to the simulating scope and different requirements, the forest fire spread simulation varies in algorithm. At present, Boundary interpolation algorithm, Boundary extension algorithm and Maze Algorithm apply to Wang Zhengfei model and its correction model. Most of the researchers use Boundary extension algorithm and Maze Algorithm, because their results are the same and also close to the actual fire spreading process. However, their calculation is very large. If they are used for a large-scale forest fire spread simulation, it will spend a lot of time. So, they are unfit for practical application. Boundary interpolation algorithm simplifies the process of forest fire spread, is of small calculation and can obtain the scope and trends of forest fire spread quickly, but accuracy is not very high. So, this paper optimizes the interpolating points and interpolation method of Boundary interpolation algorithm partially, and compares the optimized results.

\section{Reference model}

This paper mainly refers to component model of Mao Xianmin and Wang Zhengfei(Wang et al., 1983), and its algorithm lists as follows:

$\mathrm{R}_{0}=0.0299 T+0.047 W+0.009(100-h)-0.304$

$\mathrm{R}_{\text {upslope }}=R_{0} * K_{s}^{*} \operatorname{EXP}\left[3.533(\operatorname{tg} \varnothing)^{1.2}\right] * \operatorname{EXP}\left[0.1783 V^{*} \cos \theta\right]$

$\mathrm{R}_{\mathrm{downhill}}=R_{0} * K_{s} * \operatorname{EXP}\left[-3.533(\operatorname{tg} \varnothing)^{1.2}\right] * \operatorname{EXP}\left[0.1783 V^{*} \cos \left(180^{\circ}-\theta\right)\right]$

$\mathrm{R}_{\text {left-flat-slope }}=R_{0} * K_{s}^{*} \operatorname{EXP}\left[0.1783 V^{*} \cos \left(\theta+90^{\circ}\right)\right]$

$\mathrm{R}_{\text {right-flat-slope }}=R_{0} * K_{s} * * \operatorname{EXP}\left[0.1783 V^{*} \cos \left(\theta-90^{\circ}\right]\right.$

$\mathrm{R}_{\text {wind }}=R_{0}^{*} K_{s}^{*} \operatorname{EXP}\left[3.5333\left(\operatorname{tg}\left(\emptyset^{*} \cos \theta\right)\right)^{1.2}\right] * \operatorname{EXP}[0.1783 V]$

When $\theta=0^{\circ} \sim 90^{\circ}$ or $\theta=270^{\circ} \sim 360^{\circ}$

$\mathrm{R}_{\text {wind } 2}=R_{0} * K_{s}^{*} \operatorname{EXP}\left[-3.5333\left(\operatorname{tg}\left(\emptyset^{*} \cos \left(180^{\circ}-\theta\right)\right)\right)^{1.2}\right] * \operatorname{EXP}[0.1783 V]$

when $\theta=90^{\circ} \sim 270^{\circ}$

$R_{0}$ is the initial speed, $W$ is the wind at noon on the firing day, $T$ is the maximum temperature of the firing day, $\mathrm{h}$ is the minimum relative humidity. If the wind is upward, $R_{\text {wind } 1}$ will be selected. When the wind is downward, the speed will be calculated by $R_{\text {wind2 }}$.

\section{BOUNDARY INTERPOLATION ALGORITHM}

\section{A. Basal principle}

The traditional Boundary interpolation algorithm is based on grid, takes the eight-direction spreading end points of the starting fire points as sample points, and then use suitable interpolation methods to get the boundaries of the forest fire spread. Sample points are obtained by the eight-direction 
search algorithm. The principle is that only consider the eight irections are due north, northeast, due east, southeast, due south, southwest, due west and northwest, respectively. In every direction only calculate the grid on the eight directions setting out the fire point, and others are cancelled. Give the simulation time, and then when the cumulative time in all

\section{B. Choose sample points}

In accordance with the basic principle of the boundary interpolation algorithm, in a certain moment only eight spread endpoints can be obtained. But using the eight points for spatial interpolation, it is difficult to obtain fairly accurate fire boundary. Generally speaking, forest fire spreading is a gradual transfer process: the fire points of a given time $\mathrm{T}$, are formed from the fire points adjacent to the former moment $\mathrm{T}-\mathrm{t}_{1}$; the fire points of the latter moment $\mathrm{T}-\mathrm{t}_{2}$, are also formed from the points at the former moment $\mathrm{T}$, and have directive touch with $\mathrm{T}$. in the light of the principle of the forest fire spread, this paper selects the spreading endpoints at $\mathrm{T}, \mathrm{T}-\mathrm{t}, \mathrm{T}+\mathrm{t}$ as the sample points. Interval time $t$ is determined by spreading simulation time $\mathrm{T}$, which is between $\mathrm{T} / 10 \sim \mathrm{T} / 2$ to make sure the spreading endpoints of the three moments $\mathrm{T}, \mathrm{T}-\mathrm{t}, \mathrm{T}+\mathrm{t}$ have the optimal relevance.

\section{RESULTS OF THE SIMULATION}

In this paper, a forest fire that happened on April 27, 2009 at Yinanhe forest farm of Zhanhe forestry in Heilongjiang directions that the initial fire points start from. The eight $\mathrm{d}$ directions is greater than or equal to the given time, record the position of the final grid in each direction. In every direction the position of the final grid and the initial fire point are the interpolated sample points (Zhu et al., 1994; Yuan et al., 1995).

\section{Choose interpolation method}

Choosing an appropriate spatial interpolation method is a very important link to improve boundary interpolation results. There are many types of spatial interpolation, divided into global interpolation and local interpolation. The global interpolation contains trend surface model and regression model; the local interpolation contains Thiessen polygon, Density estimation, Inverse distance weighting, Thin plate spline and Kriging (Kang-tsung Chang et al., 2002). Considering that forest fire spread is a gradual process, which means that adjacent points have close touch with each other and distant points are not, this paper selects several spatial interpolation methods in local interpolation, including Inverse distance weighting, Thin plate spline and Kriging. The three interpolation methods have its own advantages and are suitable for boundary interpolation. In order to choose the most appropriate interpolation methods, this paper uses the three interpolation methods to extract the forest fire spreading boundary and compare them.

Province was regarded as the sample for the simulation. With the optimized algorithm above, we simulated the fire in details. The simulation results are as follows:

\section{A. The simulation results for before and after optimization}

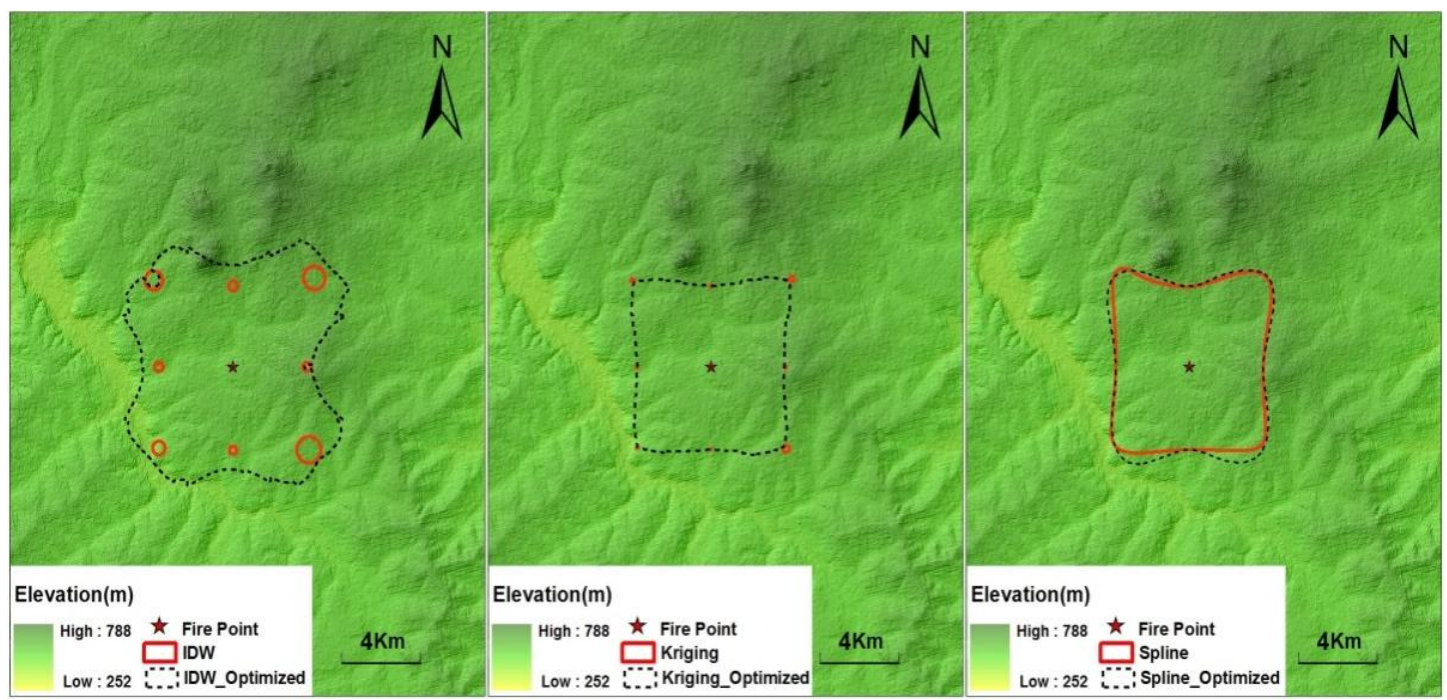

Figure 1: the results of three interpolations

Through comparing interpolation results before and after optimization (Figure 1), it is known that if only choose a single moment spread point, we cannot obtain complete forest fire spread boundary. But Simple spline interpolation could get a relatively smooth simulation context; if choose the three moments spread point after optimization, this three 
interpolation methods can get the basic scope and simulation context of forest fire spread, but the simple spline interpolation simulating results don't have big differences before and after optimization, only the spreading scope after optimization

B. The comparison between the interpolation results after optimization and the actual fire

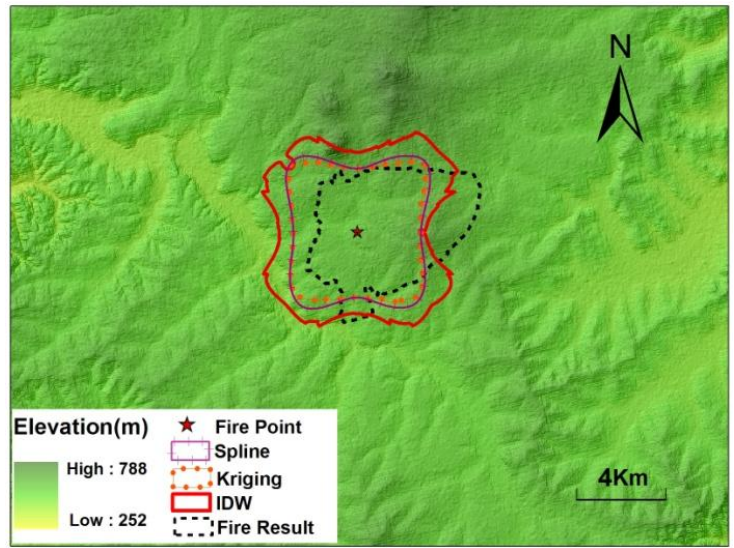

Figure 2: The results for three interpolation and actual fire range

TABLE 1: STATISTICAL DATA OF SIMULATION

\begin{tabular}{lllll}
\hline Interpolation & $\begin{array}{l}\text { Area } \\
\left(\mathrm{A}-\mathrm{km}^{2}\right)\end{array}$ & $\begin{array}{l}\text { Overlap } \\
\text { area } \\
\left(\mathrm{O}-\mathrm{km}^{2}\right)\end{array}$ & $\begin{array}{l}\text { Rate } \\
\text { of overlap } \\
\left(\mathrm{R}_{\mathrm{o}}-\%\right)\end{array}$ & $\begin{array}{l}\text { Rate } \\
\text { of fire coverage } \\
\left(\mathrm{R}_{\mathrm{f}}-\%\right)\end{array}$ \\
\hline Fire result & 58.6599 & - & - & - \\
Spline & 65.3947 & 43.1589 & 66.00 & 73.57 \\
Kriging & 59.7302 & 42.0135 & 70.34 & 71.62 \\
IDW & 98.1727 & 48.6236 & 49.53 & 82.89 \\
\hline
\end{tabular}

(Overlap area: it is the overlap area of simulation result and actual fire result Rate of overlap: $\mathrm{R}_{\mathrm{o}}=\mathrm{O}_{s} / \mathrm{A}_{\mathrm{s}}$, the rate of overlap area and simulation area

Rate of fire coverage: $\mathrm{R}_{\mathrm{f}}=\mathrm{O}_{\mathrm{s}} / \mathrm{A}_{\mathrm{f}}$, the rate of overlap area and actual fire area)

After superposing the interpolation results and the actual fire spreading scope, we found that the spreading simulating scope of Inverse Distance Weighted is bigger than the one of Simple spline interpolation and Kriging interpolation; the simulate spread scope and trend of the latter two are extremely similar, and moreover the difference between the two methods and Inverse Distance Weighted; after comparing the actual superposed fire sites, Inverse Distance Weighted covers the largest the actual fire range, and ascertains the fire spreading area to the hilt.

\section{RESULTS ANALYSIS}

Boundary interpolation algorithm after optimization mainly uses the actual forest fire spreading process to choose three adjacent moment spreading points as the boundary interpolation points, and after contrastive analysis, Inverse becoming larger a little. This shows that selecting three moments spread points could increase the channels to get the fire scope.

Distance Weighted is chosen as the spreading boundary interpolation points. Compared with the traditional Boundary interpolation algorithm, optimized algorithms not only increase the methods to obtain spreading scope, but improve the simulating precision. If we want to simulate complex forest fire quickly and accurately, optimized Boundary interpolation algorithm has great practical. Undoubtedly, optimized algorithms still require more experimental data to be validated and advised.

\section{REFERENCES}

[1] ZHAO Liang, LIU Peng-ju, ZHOU Yu-fei, SHI Jun-nan, TANG Xiao-ming. interpolation over complex terrain and its application in the simulation of forest fire spreading[J]. Journal Of Beijing Forestry University, 2010, pp,12-13.

[2] Rothermel R. C. A mathematical model for predicting fire spread in wildland fuels [J]. USDA Forest Service Research Paper, 1972: 115.

[3] Bak P., Tang C., Wiesenfeld K. Self-Organized Criticality: An Explanation of 1/f Noise [J]. Phys.Rev.Lett. 1987:381-384

[4] Karafyllidis I, Thanailakis A. A model for predicting forest fire spreading using Cellular automata. Ecological Modeling. 1997:87-97

[5] Barros F J, Mendes M T. Forest fire modeling and simulation in the DELTA environment. Simulation Practice and Theory. 1997:185-197

[6] Tang Xiaoyan, Meng Xianyu, Yi Haoruo. Review and prospect of researches on forest fire spreading models and simulating method [J]. Journal of Beijing Forestry University, 2002: 87 91

[7] Wang Zhengfei. The measurement method of the wildfire initial spread rate $[\mathrm{J}]$. Forest Science Academy of Heilongiiang. 1983:42-51

[8] Zhu J.P., Wang H.H., Wei Jing, Wang Q.A., Shao. A J. Mathematical Model for Estimating Surface Fire Behavior, 1994:33-41

[9] Yuan Hongyong, Fan Weicheng, Wang Qingan. The Mathematic Model for Surveying Sruface Fire Behovior By Use of Aerophotogrammetry and DTM[J]. Fire Safety Science, 1995:31-37

[10] Kang-tsung Chang. Introduction to Geographic Information Systems [M]. 2011:310-330 